\title{
ON THE NATURE OF THE BOUNDARY LAYER NEAR THE LEADING EDGE OF A FLAT PLATE*
}

BY
G. F. CARRIER (Brown University) AND C.
C. LIN (Massachusetts Institute of Technology)

1. Introduction. The customary treatment of boundary layer flow ${ }^{1}$ furnishes what is essentially an asymptotic expansion of the stream function (or an equivalent function defining the velocities). This solution is valid, of course, only in a region far downstream of the leading edge of the obstacle. Since one knows, however, that to all practical purposes a potential flow exists sufficiently far from the obstacle, there remains only a closed region in the neighborhood of the origin in which the nature of the flow is not known. It is the purpose of the present paper to show the nature of the flow of an incompressible fluid in this region when the obstacle is a flat plate.

2. General development. The equations governing an incompressible, viscous, fluid flow are, in conventional notation ${ }^{2}$,

$$
\begin{gathered}
u_{1} \frac{\partial u_{1}}{\partial x_{1}}+v_{1} \frac{\partial u_{1}}{\partial y_{1}}+\left(\frac{1}{\rho}\right) \frac{\partial p_{1}}{\partial x_{1}}=\nu \Delta u_{1} \\
u_{1} \frac{\partial v_{1}}{\partial x_{1}}+v_{1} \frac{\partial v_{1}}{\partial y_{1}}+\left(\frac{1}{\rho}\right) \frac{\partial p_{1}}{\partial y_{1}}=\nu \Delta v_{1} \\
\frac{\partial u_{1}}{\partial x_{1}}+\frac{\partial v_{1}}{\partial y_{1}}=0 .
\end{gathered}
$$

These may be reduced to a single equation in one unknown function in any of several ways. For convenience, we first introduce the following changes of variable: $p=$ $p_{1} / \rho u_{0}^{2}, x=x_{1} u_{0} / \nu, y=y_{1} u_{0} / \nu, u=u_{1} / u_{0}=\partial \psi / \partial y, v=v_{1} / u_{0}=-\partial \psi / \partial x, x+i y=$ $z=r e^{i \theta}$. Here, $u_{0}$ is the free stream velocity. With the introduction of these variables, Eqs. (1), (2), (3), are readily reduced to the form

$$
\partial^{4} \psi / \partial z^{2} \partial \bar{z}^{2}=\frac{1}{2 i}\left[\partial \psi / \partial \bar{z} \partial^{3} \psi / \partial z^{2} \partial \bar{z}-\partial \psi / \partial z \partial^{3} \psi / \partial z \partial \bar{z}^{2}\right] .
$$

Here $\bar{z}$ is the complex conjugate of $z$ and, we might note now, $u+i v=-2 i \partial \psi / \partial \bar{z}$.

If we restrict our analysis to the flow past a flat plate such that as $x \rightarrow-\infty, \psi \rightarrow y$, we must impose the boundary conditions $\partial \psi / \partial \bar{z} \equiv 0$ when $\theta=\cdot 0$ and when $\theta=2 \pi$. Furthermore, we must anticipate that the line $\theta=0$ will be a branch line of our solution since the higher derivatives of $\psi$ will be discontinuous along this line. We may construct such a function which is a solution of Eq. (4) in the following manner. We note that

$$
\begin{aligned}
\psi_{0} & =A\left[\bar{z} z^{1 / 2}+z \bar{z}^{1 / 2}-z^{3 / 2}-\bar{z}^{3 / 2}\right] \\
& =A r^{3 / 2}\left(\cos \frac{\theta}{2}-\cos \frac{3 \theta}{2}\right)
\end{aligned}
$$

*Received Nov. 17, 1947.

'See, for instance, L. Prandtl, The mechanics of viscous fluids, in vol. 3 of W. F. Durand's Aerodynamic theory, p. 34, Springer, Berlin, 1934.

2The subscript unity is adopted so that we may use the variable without subscripts for the quantities to be introduced later. 
is a biharmonic function for which $\theta=0$ is a branch line, along which the velocity $\partial \psi / \partial \bar{z}$ vanishes. If we write Eq. (4) in the form

$$
L(\psi)=L^{*}(\psi),
$$

where $L$ is the biharmonic operator and $L^{*}(\psi)$ is the rest of Eq. (4), then'we may define

$$
\begin{aligned}
& L\left(\psi_{0}\right)=0, \\
& L\left(\psi_{1}\right)=L^{*}\left(\psi_{0}\right), \\
& L\left(\psi_{n}\right)=L^{*}\left(\psi_{0}+\cdots+\psi_{n-1}\right)-L^{*}\left(\psi_{0}+\cdots+\psi_{n-2}\right), \\
& \cdots .
\end{aligned}
$$

Over any region in which the series

$$
\psi=\psi_{0}+\psi_{1}+\cdots
$$

converges then, we may take $\psi$ (as defined above) as a solution to Eq. (4). ${ }^{3}$ In this case, a particular integral for $\psi_{1}$ is

$$
\psi_{1}^{(1)}=A^{2} r^{3}[(\sin 2 \theta) / 40-(\theta \cos \theta / 64],
$$

and a biharmonic function which when added to $\psi_{1}^{(1)}$ adjusts the conditions at the plate, is

$$
\begin{aligned}
\psi_{1}^{(2)}=\frac{A^{2}}{128} r^{3} & {[\ln r(3 \sin \theta-\sin 3 \theta)+\theta(3 \cos \theta-\cos 3 \theta)} \\
- & \left.\frac{A^{2} r^{3}}{20}(\alpha \sin \theta+\beta \sin 3 \theta)\right],
\end{aligned}
$$

where $\alpha+3 \beta=1$. As we shall see later, $\psi_{1}^{(2)} \ll \psi_{0}$ in the matching region so that the choice $\alpha=1$ serves our purpose. The function $\psi_{1}$ becomes then

$$
\begin{aligned}
\psi_{1}=\psi_{1}^{(1)}+\psi_{1}^{(2)}=\left[A^{2} r^{3} / 128\right][\theta(\cos \theta-\cos 3 \theta) & +\ln r(3 \sin \theta-\sin 3 \theta)] \\
& +\left[A^{2} r^{3} / 40\right][\sin 2 \theta-2 \sin \theta] .
\end{aligned}
$$

It is fairly evident that the higher approximations will contain terms in $r^{9 / 2}, r^{12}$, $r^{9 / 2} \ln r, \cdots$, and will also involve coefficients with higher powers of $A$. As we (again) shall see later, $A=.083$. Thus, it is evident that within some circle this development is convergent and represents some flow. It is not immediately evident that the expansion for $\psi$ converges for $y \ll 1, x>1$. However, since our solution is of the Stokes type, it is to be anticipated that the leading term of the expansion describes the flow in a sufficiently thin neighborhood of the plate. Since we do not need to establish such a region of convergence, however, we shall not pursue the question further. ${ }^{4}$

3. The matching procedure. The solution obtained by Blasius for the velocity profile in the downstream region is usually presented in the form

${ }^{3}$ This implies that we base the expansion on the Stokes type slow-flow solutions.

${ }^{4}$ We note, however, that the choice of $\alpha$ for Eq. (10) and the corresponding "arbitrary" coefficients in $\psi_{n}$, where $n>1$, would be governed largely by this convergence consideration. 


$$
u=f^{\prime}(\eta), \quad v=\frac{1}{2(x)^{1 / 2}}\left[\eta f^{\prime}(\eta)-f(\eta)\right],
$$

where, in our notation, $\eta=y / x^{1 / 2}$. In Eqs. $(12), f(\eta)$ can be given by a power series of the form ${ }^{1}$

$$
f=\alpha \eta^{2} / 2 !-\alpha^{2} \eta^{5} / 2 \cdot 5 !+\cdots,
$$

where $\alpha=.332$.

Thus, $u=\alpha \eta-\alpha^{2} \eta^{4} / 2 \cdot 4 !=\alpha r^{1 / 2} \sin \theta /(\cos \theta)^{1 / 2}$

$$
-\alpha^{2} r^{2} \sin ^{4} \theta / 48 \cos ^{2} \theta+\cdots \text {. }
$$

That is

$$
u \simeq \alpha r^{1 / 2} \theta-\alpha^{2} r^{2} \theta^{4} / 48+\cdots
$$

in the neighborhood of $\theta=0$.

The solution of the preceding section implies that the velocity component $u$ (i.e. $\operatorname{Re}(-2 i \partial \psi / \partial \bar{z})$ be given by

$$
u=A r^{1 / 2}(5 \sin \theta / 2+\sin 3 \theta / 2)+\cdots
$$

or

$$
u \simeq 4 A r^{1 / 2} \theta+\gamma r^{2} \theta^{4}+\delta r^{2} \ln r \theta^{4}+\cdots .
$$

Two facts are evident. If these functions are to represent the same phenomenon in any overlapping regions where $r$ is not large, then $4 A \equiv \alpha$. Furthermore, we note that these two functions can never be identically the same since the $r^{2} \ln r$ term is lost in the asymptotic representation. The latter fact is quite in order since neither solution has the pretense of being a complete solution. Therefore, we may establish validity of the leading term of this representation (i.e. $\psi_{0}$ ) if we can find a region in which both $\psi_{0}$ and $f$ are valid approximations to the rigorous solution. That is, we must find a region in which $L(\psi)-L^{*}(\psi) \ll \partial^{4} \psi / \partial r^{4}$ (say). Our method of solution and its results indicate that $r \simeq 1, \theta \ll 1$ is such a region. But for $\theta$ sufficiently small and $r$ of order unity, $x^{1 / 2} f(\eta)$ is almost precisely the same function as $\psi_{0}$ (see Eq. (15)) and hence is as valid a solution. This, of course, can be worked out by computing $L\left[x^{1 / 2} f(\eta)\right]-L^{*}\left[x^{1 / 2} f(\eta)\right]$, but this is really not necessary. Hence, we have that in the neighborhood of $r=0$, Eq. (5) demonstrates the năture of the flow field provided $A=.083$. Figure 1 shows the four regions into which the flow field may be divided and the solution applicable to each. In region IV, it is doubtful if any reasonably simple analytic expression could be found for $\psi$. However, it is suggested that the flow field could be computed if one combined some intelligent "first guessing" (interpolation essentially) with the relaxation procedure and applied these to the region IV.

4. A modified Blasius solution. The alternative procedure ${ }^{5}$ for developing the foregoing solution leads to a slightly modified boundary layer solution which establishes more clearly the overlap of the slow-flow solution (which includes the leading edge) and the boundary layer solution.

\footnotetext{
${ }^{5}$ Actually, the two authors arrived at the solution independently by the two procedures described here, but felt that a joint presentation provided the most lucid account of the matter.
} 


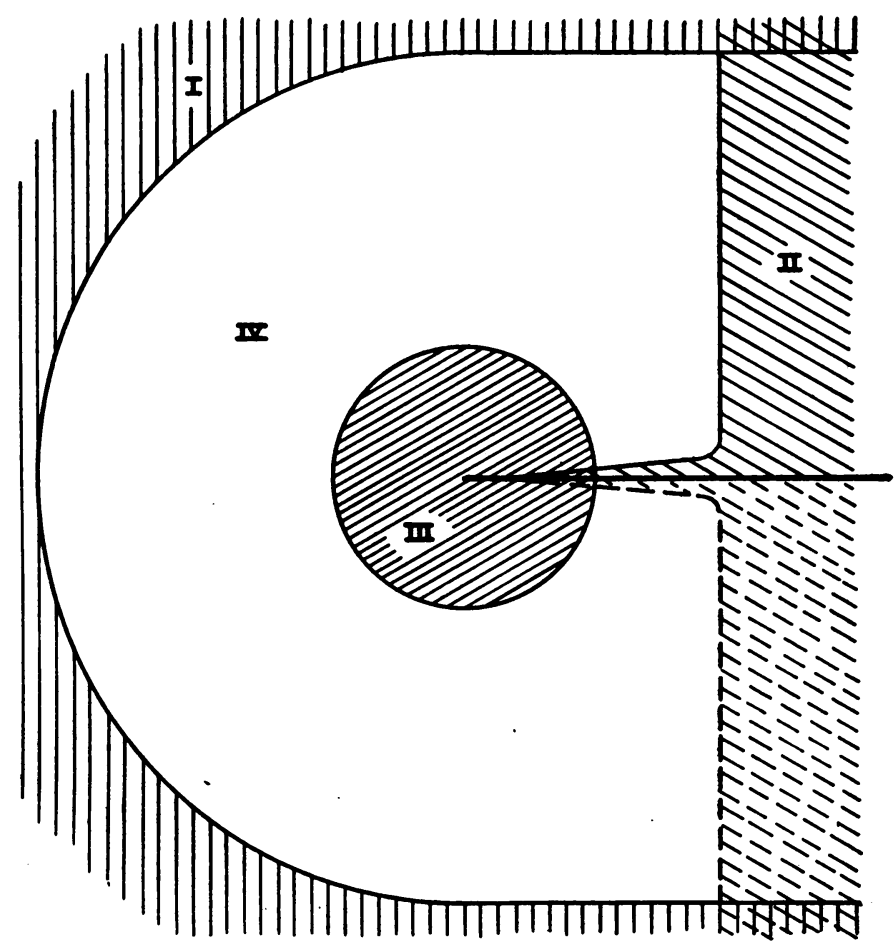

Fig. 1.
(I) Potential Solution
(II) Blasius Solution
(III) Slow Flow Solution

It seems that the most natural coordinate system to use for the flow past a flat plate is the parabolic coordinate system $(\xi, \eta)$ defined by

or

$$
x+i y=(\xi+i \eta)^{2}
$$

$$
\xi+i \eta=r^{1 / 2} \exp (i \theta / 2)
$$

By using this system of coordinates, it is possible to give a unified discussion of the boundary layer solution and the slow motion solution in the neighborhood of the plate, including the leading edge.

In the parabolic system of coordinates, the equation for the stream function $\psi$ becomes

where

$$
L(\psi)=\left(\xi^{2}+\eta^{2}\right)\left(\psi_{\xi} \Delta \psi_{\eta}-\psi_{\eta} \Delta \psi_{\xi}\right)-2\left(\eta \psi_{\xi}-\xi \psi_{\eta}\right) \Delta \psi,
$$

$$
L(\psi) \equiv\left(\xi^{2}+\eta^{2}\right) \Delta \Delta \psi-4\left(\xi \Delta \psi_{\xi}+\eta \Delta \psi_{\eta}-\Delta \psi\right) .
$$

From a consideration of the diffusive effect of the viscous forces, it is obvious that the effect of viscosity is essentially limited to regions with $\eta \sim 1$, close to the solid boundary. For very small values of $\eta$, the motion is slow. Since $\psi=0$ and $\partial \psi / \partial \eta=0$ at $\eta=0$ it is obvious that in this region a good approximation to (18) is

$$
L(\psi)=0 .
$$


A polynomial solution satisfying the boundary conditions $\psi=\psi_{\eta}=0$ at $\eta=0$ is

$$
\psi=A \xi \eta^{2}
$$

which agrees with Eq. (5) of Sec. 2. This solution can also be obtained from Eq. (19) by writing $\psi=\eta^{2}(f(\xi)+0(\eta))$ for small $\eta$.

If $\xi$ is large while $\eta \sim 1$, one may introduce a new variable

$$
\xi^{\prime}=\epsilon \xi
$$

where $\epsilon$ is a small parameter. ${ }^{6}$ In the limit $\epsilon \rightarrow 0$, the equation (3) becomes

$$
\xi^{\prime 2} \frac{\partial^{4} \psi^{(0)}}{\partial \eta^{4}}=\xi^{\prime 2}\left(\psi_{\xi^{\prime}}^{(0)} \psi_{\eta^{2}}^{(0)}-\psi_{\eta}^{(0)} \psi_{\xi^{\prime} \eta^{2}}^{(0)}\right)+2 \xi^{\prime} \psi_{\eta}^{(0)} \psi_{\eta^{2}}^{(0)}
$$

where

$$
\psi^{(0)}=\epsilon \psi
$$

This equation may be solved by putting

$$
\psi^{(0)}=\xi^{\prime} f(\eta) ;
$$

then $f(\eta)$ satisfies

$$
f^{i v}=f f^{\prime \prime \prime}+f^{\prime} f^{\prime \prime},
$$

or

$$
f^{\prime \prime \prime}=f f^{\prime \prime}
$$

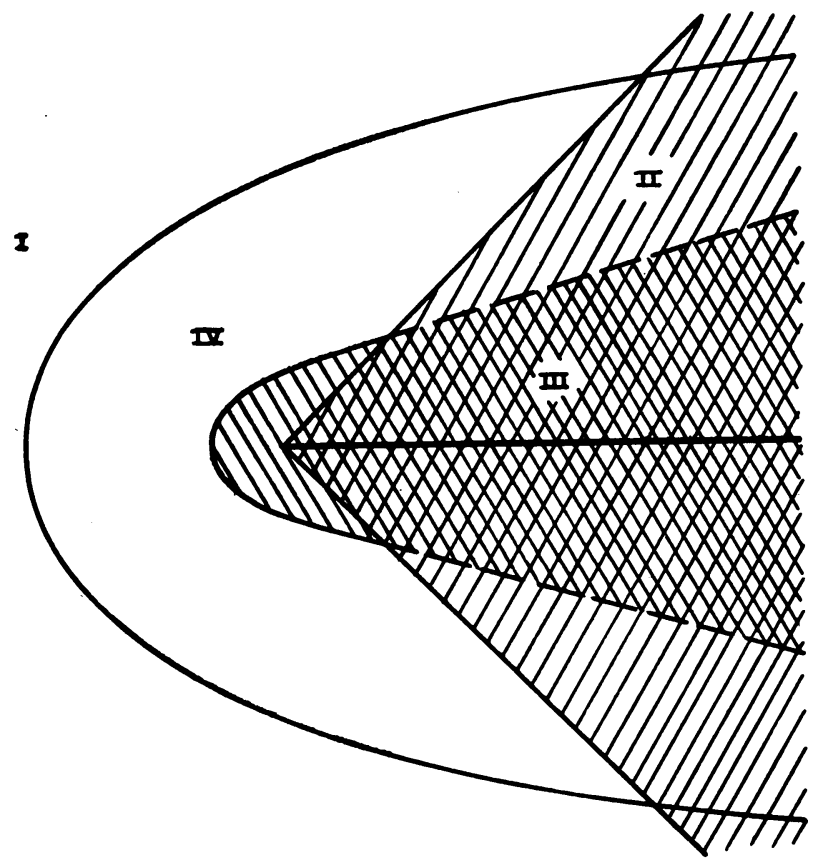

Fig. 2.

(I) Potential Solution

(II) Modified Blasius Solution

(III) Slow Flow Solution

'To give $\epsilon$ an explicit meaning, we consider the region $\eta \leq \epsilon \xi$. 
this is the usual boundary layer equation except that $\eta$ is not quite the conventional parameter. ${ }^{7}$ Higher approximations may be obtained by solving (18) in powers of $\epsilon$.

Thus, the regions of approximate validity of these solutions are as follows:

(i) potential solution: $\quad \eta \gg 1$;

(ii) boundary layer equation: $\eta \sim 1, \eta \ll \xi$;

(iii) Stokes equation: $\quad \eta \ll 1$, irrespective of $\xi$.

These regions are indicated in Fig. 2. It is obvious that there is an overlap of regions (ii) and (iii) where the solution (5) agrees with the boundary layer solution. Again, we have not established the convergence of the slow flow solution. However, the physical considerations which lead to the slow flow and the boundary layer solutions indicate that they are at least valid asymptotic approximations, if not convergent processes. This is further strengthened by the identical nature of the leading terms of the two types of solutions for $\eta \ll 1$.

${ }^{7}$ Here, $\eta=r^{1 / 2} \sin \theta / 2$, conventionally $\eta=r^{1 / 2} \sin \theta /(\cos \theta)^{1 / 2}$. 